\title{
In memoriam Claus Buddeberg
}

Am 2. Februar 2017 ist Claus Buddeberg verstorben. Claus Buddeberg hat das Gebiet der Psychosomatik und der Sexualmedizin in der Schweiz und im deutschsprachigen Raum über lange Jahre intensiv mitgeprägt und mitgestaltet.

Claus Buddeberg wurde 1946 in Säckingen nahe an der Schweizer Grenze als Sohn eines Hausarztes geboren. Claus begleitete seinen Vater häufig auf seinen Krankenbesuchen in die umliegenden Dörfer. Entgegen den familiären Erwartungen wurde Claus nicht Hausarzt, sondern Psychiater. Neben dem Medizinstudium interessierten ihn immer auch soziologische Vorlesungen. Die Studienjahre verbrachte er in Tübingen, Lausanne und Hamburg, und seine Facharztausbildung begann er am Burghölzli. Zusammen mit seiner Frau Barbara, welche er im Studium kennengelernt hatte, übersiedelte er 1973 nach Zürich, wo er nach weiteren Assistenzarztjahren am UniversitätsSpital auf der Neurologie und an der Psychiatrischen Poliklinik junger Oberarzt auf der Psychotherapiestation wurde. Gemeinsam mit Jürg Willi und Edgar Heim entwickelte er das neue Studienfach Psychosoziale Medizin, welches den Medizinstudenten in der Schweiz erstmals vertieft die sozialen und ethischen Aspekte sowie die Grund-

\section{Gemeinsam mit Jürg Willi und \\ Edgar Heim entwickelte er das neue \\ Studienfach Psychosoziale Medizin.}

lagen der Kommunikation in der Medizin vermittelte. Er war einer der Ersten, die mit Videoanalysen arbeiteten und auch andere neue Medien in den Unterricht einführten.

1983 habilitierte er und baute im UniversitätsSpital Zürich die Konsiliar- und Liaisonpsychiatrie auf. Er war als Konsiliararzt für seine konzisen psychiatrischen Beurteilungen und seine engagierte $\mathrm{Zu}$ sammenarbeit sehr geschätzt. Eine gute Patientenversorgung war ihm stets ein grosses Anliegen.

Mit grossem Elan initiierte Claus Buddeberg nach einem Aufenthalt in den USA ein Kompetenzzentrum für psychosoziale Forschung und leitete diverse Forschungsprojekte zu interdisziplinären Fragestellungen bei Krebserkrankungen, in der Transplantations- und Sexualmedizin. Ein grosses Anliegen war ihm auch die Förderung des akademischen Nachwuchses, den er durch seine strukturierte Arbeitsweise und durch die

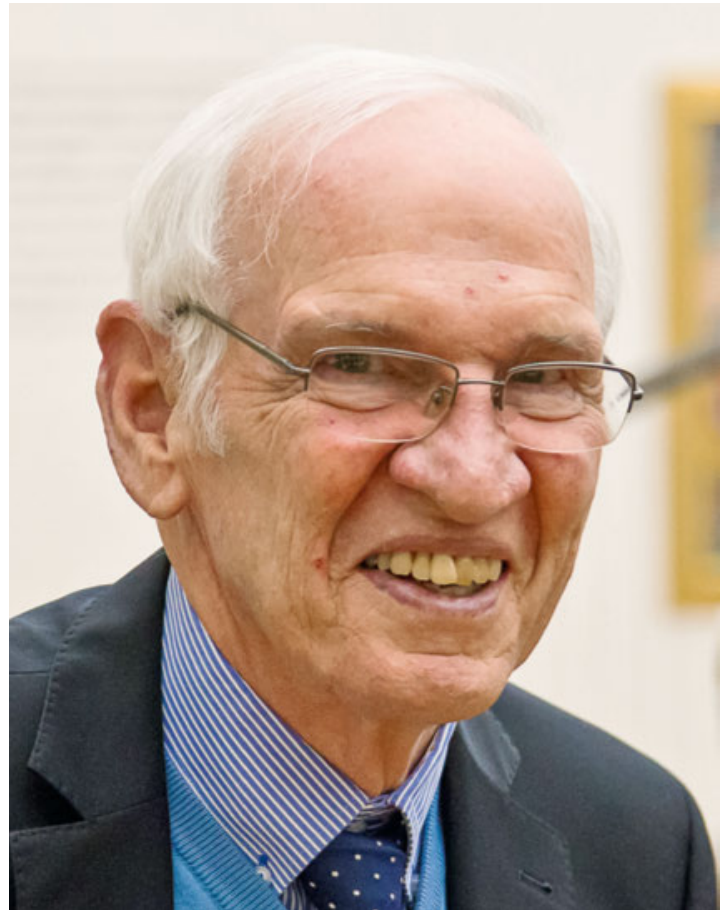

Claus Buddeberg (1946-2017).

Vermittlung internationaler Kontakte in sein wissenschaftliches Kompetenzfeld einführte und fachlich und menschlich prägte.

Für die Lehre und die Forschung auf dem Gebiet der Psychosomatik kooperierte Claus Buddeberg mit anderen humanwissenschaftlichen Fachgebieten wie der biomedizinischen Ethik, der Sozial- und Präventivmedizin. Ausgehend von diesem interdisziplinären Ansatz entwickelte er zusammen mit seinen Mitarbeitern einen 2-jährigen berufsbegleitenden Kurs in Psychosomatischer und Psychosozialer Medizin für Haus-

\section{Mit grossem Elan initiierte Claus Buddeberg} ein Kompetenzzentrum für psychosoziale Forschung.

ärzte, Gynäkologinnen, Rheumatologen und andere Grundversorger. Daraus entstand das Ausbildungsinstitut IHM und in Zusammenarbeit mit der Universität Zürich ein CAS-Studiengang, dessen erster Direktor er wurde. Auf gesamtschweizerischer Ebene war er Mitbegründer der Schweizerischen Akademie für Psychosoziale und Psychosomatische Medizin SAPPM. Als wichtiger Wegbereiter für die Psychosomatik in der 
Schweiz brachte Claus Buddeberg seine standespolitischen und fachlichen Kenntnisse in diese Dachorganisation ein, bis zuletzt als Mitglied des wissenschaftlichen Beirats.

Neben seinen Aufgaben als Lehrstuhlinhaber für psychosoziale Medizin und als Leiter der Abteilung für Psychosoziale Medizin am UniversitätsSpital Zürich übernahm Claus Buddeberg 2001 die Leitung der neu eröffneten Abteilung für Psychosomatische Medizin an der Zürcher Höhenklink in Davos. Vernetzt mit der Spezialsprechstunde für psychosomatische Störungen am USZ und damit konzeptuell hoch innovativ, entwickelte er ein interdisziplinäres Behandlungsprogramm für Patientinnen und $\mathrm{Pa}-$ tienten mit chronischen Schmerzkrankheiten und anderen psychosomatischen Erkrankungen.

Claus Buddeberg war ein väterlicher Vorgesetzter, der sich nicht nur für die beruflichen Aspekte interessierte, sondern auch immer ein offenes Ohr für die privaten Anliegen und Probleme seiner Mitarbeitenden hatte. Familienfreundliche Arbeitsbedingungen waren ihm ein echtes Anliegen und waren gelebte Realität. Früher als andere schuf er für Frauen kinderfreundliche Anstellungsbedingungen und ermöglichte den jungen Vätern in seiner Abteilung, durch flexible Arbeitszeiten mehr Zeit mit ihren Kindern zu verbringen.

\section{Auf gesamtschweizerischer Ebene war er Mitbegründer der Schweizerischen Akademie für Psychosoziale und Psychosomatische Medizin SAPPM.}

Claus Buddeberg war didaktisch geschickt, konnte sein enormes Wissen klar vermitteln, und es gelang ihm, die Mitarbeitenden und die Studierenden immer wieder für das komplexe Gebiet der Psychosomatik zu begeistern. Er war auch ein origineller Denker, der sich oft schnell eine eigenständige Meinung bildete, die er meist auch hartnäckig vertrat. Es brauchte schon gute Argumente, Ausdauer und Humor, um ihn umzustimmen. So war seine Bereitschaft, sich mit seinen Mitar- beitern offen auseinanderzusetzen und seine Werte zu vertreten zwar gelegentlich etwas anstrengend, aber es waren auch Verbindlichkeit und Präsenz - Eigenschaften, die wir heute allzu oft vermissen -, die seine Persönlichkeit auszeichneten. Dazu gehörte auch der ihm eigene Humor. Mit Claus Buddeberg konnte man lachen, auch wenn das Arbeitsleben mitunter Belastendes bereithielt.

Claus konnte begeistern für spannende Projekte, für neue Gedanken, für Wagnisse, und er beteiligte sich selbst immer mit seinem vollen Elan und Einsatz daran - zuverlässig, loyal und konsequent.

Bei aller Beharrlichkeit bei der Verfolgung seiner Ziele zeichnete Claus Buddeberg aber auch die Fähigkeit aus, loszulassen, wenn er zum Schluss kam, dass er für gewisse Aufgaben nicht mehr persönlich Verantwortung tragen musste. Nach seiner Emeritierung 2010 führte er eine kleine psychotherapeutische Praxis, hatte verschiedene Supervisionsaufträge, und er stellte sich als Mentor mit seinem gesamten Wissen und seiner institutionellen Vernetzung dem Studiengang und der Schweizerischen Gesellschaft für Psychosomatische und Psychosoziale Medizin weiterhin zur Verfügung. Claus Buddebergs grosse Lebensenergie hat sich erschöpft, viel zu früh aufgezehrt durch ein schweres Leiden. Dabei war es diese grosse, ansteckende Lebensenergie und Freude, mit der er uns als Mitarbeitende und Freunde vor Jahren und über Jahre in seinen Bann gezogen hatte. Claus konnte begeistern für spannende Projekte, für neue Gedanken, für Wagnisse, und er beteiligte sich selbst immer mit seinem vollen Elan und Einsatz daran - zuverlässig, loyal und konsequent. Jetzt ist es an uns, loszulassen. Traurig bleiben wir zurück, dankbar für das gemeinsam Erlebte.

Roland von Känel, Stefan Büchi, René Pomeranz, Daniel Ackermann, Marianne Schneider 